\title{
Link availability of satellite-based FSO communications in the presence of clouds and turbulence
}

\author{
Thang V. Nguyen ${ }^{1, \text { a) }}$, Hoang D. Le ${ }^{1}$, Thanh V. Pham ${ }^{1}$, \\ and Anh T. Pham ${ }^{1}$ \\ ${ }^{1}$ Computer Communications Laboratory, The University of Aizu, \\ Tsuruga, Ikki-machi, Aizuwakamatsu, Fukushima 965-8580, Japan \\ a)d8212104@u-aizu.ac.jp
}

\begin{abstract}
This letter addresses the link availability issue in satellitebased free space optical (FSO) communications. In particular, we present an accurate analysis for the estimation of the link availability in the presence of clouds and atmospheric turbulence. For this purpose, a meteorological ERA-Interim database, produced by the European Center for Medium Range Weather Forecast (ECMWF), is investigated. The obtained data is, then, used to analyze the link availability for several regions in Japan. In addition, site diversity scheme is also investigated to enhance the system availability.
\end{abstract}

Keywords: optical satellite links, cloud attenuation, site diversity Classification: Satellite Communications

\section{References}

[1] H.D. Le, V.V. Mai, C.T. Nguyen, and A.T. Pham, "Throughput analysis of incremental redundancy hybrid ARQ for FSO-based satellite systems," IEEE Veh. Tech. Conf., pp. 1-5, 2019. DOI: DOI:10.1109/VTCFall.2019.8891163

[2] H. Kaushal and G. Kaddoum, "Optical communication in space: Challenges and mitigation techniques,” IEEE Commun. Surveys Tuts., vol. 19, no. 1, pp. 57-96, 2017. DOI: DOI:10.1109/COMST.2016.2603518

[3] K. Suzuki, D.R. Kolev, A. Carrasco-Casado, and M. Toyoshima, "Environmentaldata collection system for satellite-to-ground optical communications," Trans. JSASS Aerospace Tech. Japan, vol. 16, no. 1, pp. 35-39, 2018. DOI: DOI:10. 2322/tastj.16.35

[4] N.K. Lyras, C.N. Efrem, C.I. Kourogiorgas, and A.D. Panagopoulos, "Optimum monthly based selection of ground stations for optical satellite networks," IEEE Commun. Lett., vol. 22, no. 6, pp. 1192-1195, 2018. DOI: DOI:10.1109/ LCOMM.2018.2819174

[5] N.K. Lyras, C.N. Efrem, C.I. Kourogiorgas, A.D. Panagopoulos, and P.D. Arapoglou, "Optimizing the ground network of optical MEO satellite communication systems," Syst. J., vol. 14, no. 3, pp. 3968-3976, 2020. DOI: 10.1109/jsyst.2019.2945838

[6] H.D. Le, T.V. Nguyen, and A.T. Pham, "Cloud attenuation statistical model for satellite-based fso communications," IEEE Antennas and Wireless Propagation Letters, 2021. DOI: DOI:10.1109/LAWP.2021.3058641 
[8] D. Giggenbach and F. Moll, "Scintillation loss in optical low earth orbit data downlinks with avalanche photodiode receivers," IEEE Int. Conf. Space Opt. Syst., pp. 115-122, 2017. DOI: DOI:10.1109/ICSOS.2017.8357220

\section{Introduction}

In recent years, broadband satellite networks using free-space optical (FSO) communications have attracted considerable attention from both academia and industry thanks to the combined advantage of global coverage and huge bandwidth connectivity [1]. The primary concerns of FSO are the atmospheric turbulence and cloud blockage [2], which can severely deteriorate the system performance and link availability. For this reason, an investigation of the link availability in particular sites of interest should be performed in planning and designing a satellite-based FSO communications system.

In this regard, there have been several studies focusing on the estimation of the link availability in the presence of clouds due to its very high attenuation $[3,4,5]$. In particular, Suzuki et. al. investigated the link availability for several regions in Japan based on the image data of clouds collected by a whole-sky camera at the ground [3]. The method used in this work is, however, not always possible for other sites at different regions as additional facilities are required. Another method was based on the cloud liquid water content (CLWC) data from available databases measured by satellites $[4,5]$.

It is worth noting that most of the existing studies on link availability estimation were often based on the cloud coverage statistics. In other words, FSO links were considered to be blocked whenever there is a presence of clouds determined by the cloud image data in [3] or by CLWC $>0$ in [4, 5]. Nevertheless, as reported in our recent study [6], the FSO links can still be operational in the presence of clouds with low CLWC values. In addition, even with a small cloud attenuation, the link availability can still be seriously degraded due to the atmospheric turbulence, which was not considered in the above-mentioned works.

This letter, therefore, aims to provide a more accurate analysis for the estimation of the link availability considering the impact of both clouds and atmospheric turbulence. First, we obtain the CLWC data from the meteorological ERA Interim database produced by the European Center for Medium Range Weather Forecast (ECMWF) [7]. Based on the cloud attenuation statistical model developed in [6], the data is, then, used to derive the link availability for several regions in Japan. In addition, site diversity scheme is also investigated to improve the link availability.

\section{Network descriptions}

A satellite-based FSO network is illustrated in Fig. 1, in which we consider laser downlinks from a satellite to multiple ground stations (GSs) ${ }^{1}$. During the downlink transmission, FSO links may experience (a) cloud blockage, (b) clouds with low CLWC values that the connection is still maintained, and (c) clear sky. In the

\footnotetext{
${ }^{1}$ The optical ground stations shown in Fig. 1 are developed by NICT, Japan.
} 


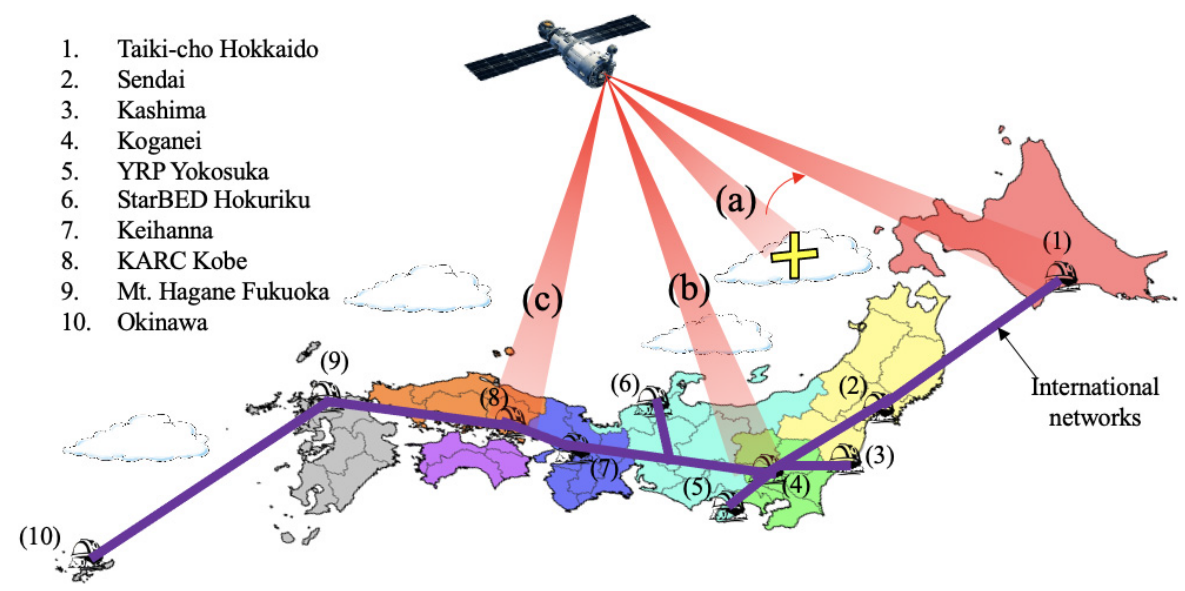

Fig. 1. Satellite downlinks with multiple ground stations.

following, we focus on the two major impairments on the FSO links, namely cloud attenuation and atmospheric turbulence.

\subsection{Cloud attenuation}

The liquid water particles in clouds cause the scattering phenomenon of the laser beam propagation, leading to the decrease of visibility and significant attenuation of signal power. Based on the CLWC data obtained from the ERA Interim database [7], the visibility $(\mathrm{km})$ can be estimated as

$$
V=\frac{1.002}{\left(L \times N_{\mathrm{c}}\right)^{0.6473}},
$$

where $L\left(\mathrm{~g} / \mathrm{m}^{3}\right)$ is the CLWC value and $N_{\mathrm{c}}\left(\mathrm{cm}^{-3}\right)$ is the cloud droplet number concentration. Using Mie theory, the visibility is then used to derive the cloud attenuation, which can be found in [6].

According to [6], the log-normal distribution can be used to accurately model the cloud attenuation whose the probability density function (PDF) is given as

$$
f_{A_{\mathrm{c}}}\left(A_{\mathrm{c}}\right)=\frac{1}{\sqrt{2 \pi} \sigma A_{\mathrm{c}}} \exp \left[-\frac{\left(\ln A_{\mathrm{c}}-\mu\right)^{2}}{2 \sigma^{2}}\right],
$$

where $A_{\mathrm{c}}$ is the cloud attenuation expressed in $\mathrm{dB}$ while $\sigma$ and $\mu$ are obtained based on the maximum likelihood estimation of the curve-fitting method.

\subsection{Atmospheric turbulence}

The atmospheric turbulence phenomenon causes the scintillation effect, which results in fluctuations in the received signal power. The turbulence strength is determined by the Rytov variance, denoted as $\sigma_{R}^{2}$, where weak, moderate and strong turbulence conditions correspond to $\sigma_{R}^{2}<1, \sigma_{R}^{2} \approx 1$ and $\sigma_{R}^{2}>1$, respectively [2]. For the satellite-to-ground optical channels, the Rytov variance in the case of plane wave propagation is given as

$$
\sigma_{R}^{2}=2.25\left(\frac{2 \pi}{\lambda}\right)^{7 / 6} \sin ^{-11 / 6}(\theta) \int_{H_{\mathrm{G}}}^{H_{\mathrm{sat}}} C_{n}^{2}(h)\left(h-H_{\mathrm{G}}\right)^{5 / 6} \mathrm{~d} h,
$$


where $\lambda(\mathrm{nm})$ is the optical wavelength, $\theta$ is the satellite elevation angle, while $H_{\mathrm{G}}$ and $H_{\text {sat }}$ are the receiver and satellite altitudes, respectively. In addition, $C_{n}^{2}(h)$ is the altitude-dependent index structure parameter, which can be found in [2].

\section{Performance analysis}

This Section focusses on an estimation of the link availability. Specifically, we first analyze the power loss due to the cloud attenuation and atmospheric turbulence. The link availability is, then, estimated based on the obtained power loss. In addition, site diversity scheme is also investigated to improve the link availability.

\subsection{Power loss}

The power loss due to the cloud attenuation and atmospheric turbulence, denoted as $A_{\text {loss }}(\mathrm{dB})$, can be expressed as

$$
A_{\text {loss }}=A_{\mathrm{c}}+A_{\mathrm{t}}
$$

where $A_{\mathrm{t}}$ is the power loss caused by the turbulence-induced fading.

Let $d_{\mathrm{fso}}$ be the distance from the satellite to the ground station, $A_{\mathrm{t}}(\mathrm{dB})$ is then given by

$$
A_{\mathrm{t}}=4.343\left[\operatorname{erfcinv}\left(2 p_{\mathrm{th}}\right) \sqrt{2 \ln \left(\sigma_{\mathrm{P}}^{2}+1\right)}-\frac{1}{2} \ln \left(\sigma_{\mathrm{P}}^{2}+1\right)\right],
$$

where $\sigma_{\mathrm{P}}^{2}=\sigma_{R}^{2}\left[1+0.33\left(\frac{\pi D^{2}}{2 \lambda d_{\mathrm{fso}}}\right)^{5 / 6}\right]^{-7 / 5}$ is the power scintillation index with $D$ being the receiver aperture diameter and $p_{\mathrm{th}}=10^{-2}$ [8]. On the other hand, the cloud attenuation $A_{\mathrm{c}}$ can be written as

$$
A_{\mathrm{c}}=\sum_{k=1}^{M} 4.343\left[\frac{3.91}{V_{k}}\left(\frac{\lambda}{550}\right)^{-\delta_{\mathrm{k}}}\right] \frac{\Delta h_{\mathrm{k}}}{\sin (\theta)},
$$

where $M$ is the total cloud layers considered while $V_{\mathrm{k}}$ and $\Delta h_{\mathrm{k}}$ are the visibility and the vertical extent of the $k$-th layer, respectively [6]. In addition, $\delta_{\mathrm{k}}$ depends on $V_{\mathrm{k}}$, which can found in [2].

\subsection{Link availability}

Given the power loss in (4), the link availability can be estimated by

$$
P_{\text {avai }}=\operatorname{Pr}\left[A_{\text {loss }} \leq A_{\text {budget }}\right]=\operatorname{Pr}\left[A_{\mathrm{c}} \leq A_{\text {budget }}-A_{\mathrm{t}}\right],
$$

where $A_{\text {budget }}(\mathrm{dB})$ is the total link budget for the cloud attenuation and turbulence loss. Based on (2) and given $A_{\mathrm{th}}=A_{\text {budget }}-A_{\mathrm{t}}(\mathrm{dB})$, (7) is rewritten as

$$
P_{\text {avai }}=\int_{0}^{A_{\text {th }}} f_{A_{\mathrm{c}}}\left(A_{\mathrm{c}}\right) \mathrm{d} A_{\mathrm{c}}=\frac{1}{2}+\frac{1}{2} \operatorname{erf}\left[\frac{\ln \left(A_{\mathrm{th}}\right)-\mu}{\sqrt{2} \sigma}\right] .
$$




\subsection{Site diversity technique}

To improve the link availability, we consider site diversity technique. Let $K$ denote the total number of sites that are available for communications. By applying the technique, the link availability, which is defined when at least one station is available at any time, can be determined as

$$
A=1-\prod_{i=1}^{K} \operatorname{Pr}\left[A_{\text {loss }}(i)>A_{\text {budget }}\right]=1-\prod_{i=1}^{K} P_{\text {non }}(i),
$$

where $P_{\text {non }}(i)=1-P_{\text {avai }}(i)$, with with $P_{\text {avai }}$ being given in (8), is the probability that the $\mathrm{i}-t h$ station is not available at a given time.

\section{Results and discussions}

In this Section, numerical results of the link availability during rainy season (i.e., July) in a 5-year period (2015-2019) for several regions in Japan are presented. The CLWC data are collected with one sample every hour over a spatial resolution of $0.25^{\circ} \times 0.25^{\circ}$ latitude/longitude grid and with $M=5$ cloud layers within $1-3 \mathrm{~km}$ above sea level. For simulations of the atmospheric turbulence, the optical wavelength of $\lambda=1550 \mathrm{~nm}$, satellite elevation angle $\theta=40^{\circ}$, satellite altitude $H_{\mathrm{sat}}=36,000 \mathrm{~km}$, receiver aperture diameter $D=10 \mathrm{~cm}$, and receiver altitude $H_{\mathrm{G}}=10 \mathrm{~m}$ are chosen.

\subsection{Impact of clouds on link availability}

Firstly, we investigate the impact of clouds without considering the atmospheric turbulence. In this scenario, Fig. 2 illustrates the link availability for 47 prefectures in Japan when the link budget for the cloud attenuation is $30 \mathrm{~dB}$. It is observed that Hokkaido and Shikoku achieve the highest and lowest levels of link availability during the rainy season, respectively. In addition, the link availability decreases from the north to the south of Japan. From the network design perspective, the obtained

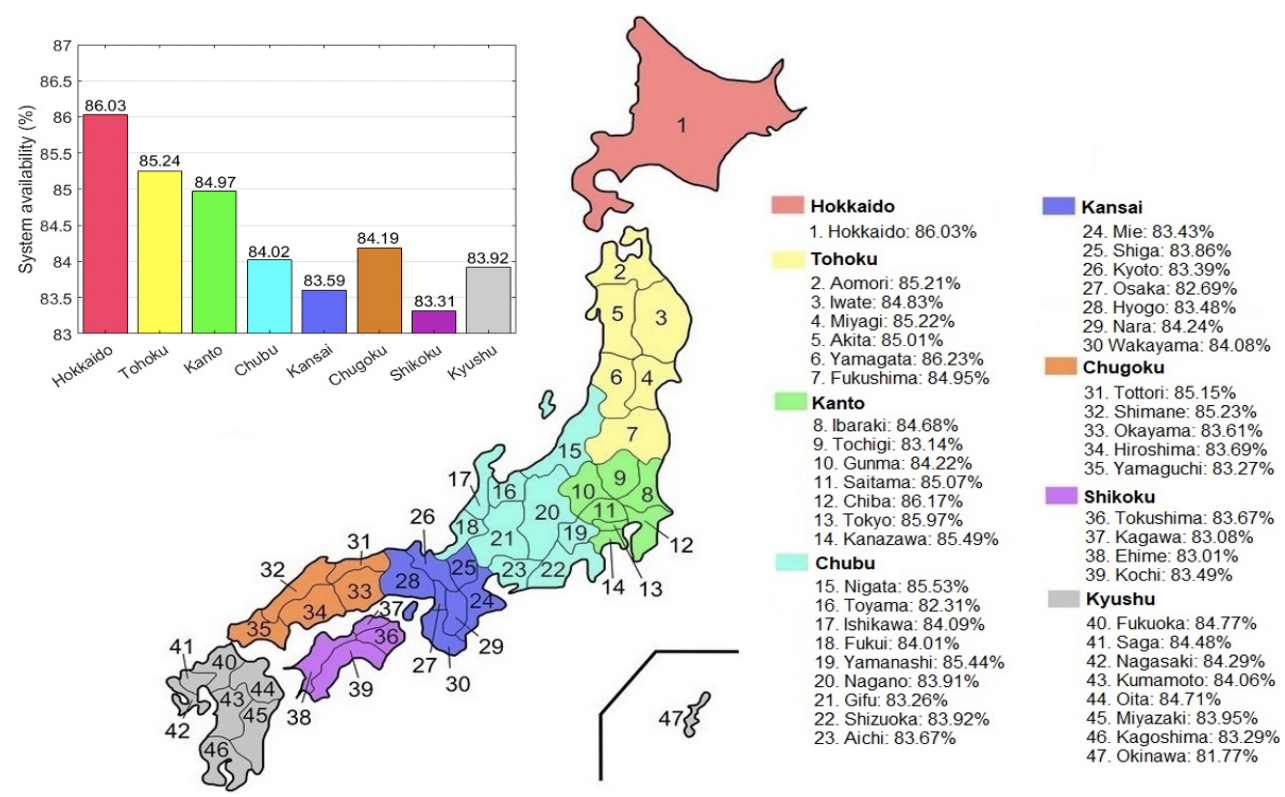

Fig. 2. Link availability in Japan during rainy season. 


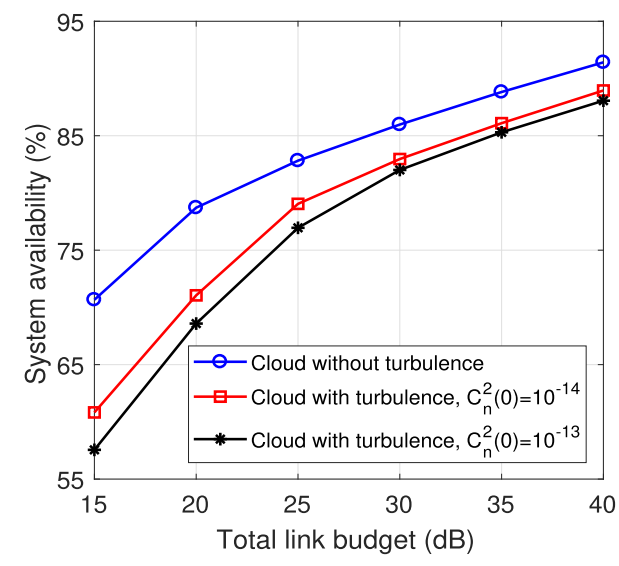

(a)

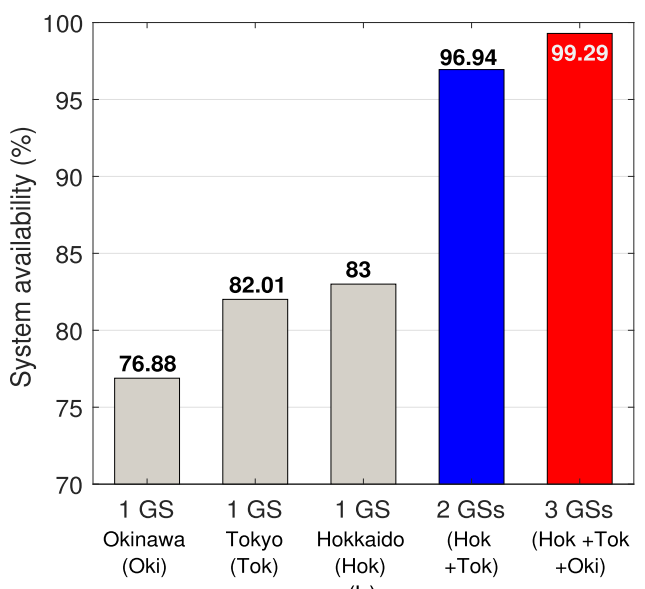

(b)

Fig. 3. Link availability (a) without site diversity, (b) with site diversity.

results are useful in deciding the optical GSs that can communicate reliably with the satellite.

\subsection{Joint impact of clouds and atmospheric turbulence}

Next, Fig. 3(a) depicts the link availability in Tokyo over a range of link budgets under the joint impact of clouds and atmospheric turbulence. Here, the total link budget, $A_{\text {budget }}$, for the cloud attenuation and turbulence loss is considered. As seen from the figure, severe clouds combined with the atmospheric turbulence significantly deteriorate the link availability. For instance, when $A_{\text {budget }}=30 \mathrm{~dB}$, the link availability are $86 \%, 83 \%$, and $82 \%$ in no turbulence, weak turbulence, and strong turbulence cases, respectively.

Finally, we highlight the advantage of site diversity technique in improving the link availability in Fig. 3(b) when $A_{\text {budget }}=30 \mathrm{~dB}$ and $C_{n}^{2}(0)=10^{-13}$. It is evident that the link availability increases with the number of GSs. For example, the link availability reaches $99.29 \%$ when using three GSs.

\section{Conclusions}

This letter has presented an estimation for the link availability of satellite-based FSO communications in the presence of clouds and atmospheric turbulence. The 5-year reanalysis meteorological ECMWF ERA Interim database for Japan was used for the investigation. Site diversity technique was employed to improve the link availability. Comprehensive numerical results were presented to show the link availability during rainy season and to confirm the effectiveness of using site diversity technique. 